2016-03

Neural connectivity of alexithymia: Specific association with major depressive disorder

\author{
Ho, Nerissa
}

http://hdl.handle.net/10026.1/16579

\begin{abstract}
10.1016/j.jad.2015.12.057
Journal of Affective Disorders

Elsevier BV
\end{abstract}

All content in PEARL is protected by copyright law. Author manuscripts are made available in accordance with publisher policies. Please cite only the published version using the details provided on the item record or document. In the absence of an open licence (e.g. Creative Commons), permissions for further reuse of content should be sought from the publisher or author. 
Research paper

\title{
Neural connectivity of alexithymia: Specific association with major depressive disorder
}

\author{
Nerissa S.P. Ho ${ }^{\mathrm{a}, \mathrm{b}}$, Michael M.C. Wong ${ }^{\mathrm{c}}$, Tatia M.C. Lee ${ }^{\mathrm{a}, \mathrm{b}, \mathrm{d}, \mathrm{e}, *}$ \\ a Laboratory of Neuropsychology, The University of Hong Kong, Pokfulam Road, Hong Kong \\ ${ }^{\mathrm{b}}$ Laboratory of Cognitive Affective Neuroscience, The University of Hong Kong, Hong Kong \\ c Department of Psychiatry, Queen Mary Hospital, Hong Kong \\ ${ }^{\mathrm{d}}$ Institute of Clinical Neuropsychology, The University of Hong Kong, Hong Kong \\ e The State Key Laboratory of Brain and Cognitive Sciences, The University of Hong Kong, Hong Kong
}

\section{A R T I C L E I N F O}

\section{Article history:}

Received 6 September 2015

Received in revised form

19 December 2015

Accepted 26 December 2015

Available online 1 January 2016

Keywords:

Alexithymia

Depression

White-matter

Resting-state

Corpus callosum

Superior longitudinal fasciculus

\begin{abstract}
A B S T R A C T
Background: Alexithymia has been frequently associated with major depression disorders (MDD). Yet little is known about the exact relationship of alexithymia and MDD. In order to explore this subject matter, the neural connectivity associated with alexithymia in people with MDD and matched nonclinical controls were compared.

Methods: Twenty-two females diagnosed with first-episode MDD and twenty-one matched nonclinical controls were MRI brain-scanned with diffusion-tensor-imaging and resting-state-functional-imaging methods, and self-reported the Chinese 20-item Toronto Alexithymia Scale.

Results: Voxel-wise multiple regression analysis showed a group interaction effect regarding the correlation between white-matter-connectivity and alexithymia. Significant correlations were observed at the corpus-callosum in MDDs and at the right superior-longitudinal-fasciculus in the controls. These findings were then used to derive seeds for analyzing resting-state-functional-connectivity in each group separately. The results further revealed that alexithymia in MDDs were associated with reduced functional-connectivity in the right precentral-gyrus and several regions of the brain on the right which are associated with cognitive regulation in the default-mode-network. In contrast, among the control subjects, alexithymia was correlated with increased functional-connectivity between the right inferiorfrontal-gyrus-triangularis and the right superior-occipital-lobe, which is associated with emotional response to external stimuli.

Limitations: Better participant selection, especially recruitment of medication-free samples, and the engagement of additional alexithymia assessments, should be considered in future investigations.

Conclusions: These findings supported our a priori hypothesis that MDDs and controls have distinct white-matter correlates of alexithymia, and these corresponded to the existing proposed neural correlates for the cognitive and affective characteristics of alexithymia respectively. Extended impacts of these microstructural changes on remote functional networks might help explain the distinct behavioral characteristics of alexithymia for these groups, as well as implications for therapeutic intervention of MDD.
\end{abstract}

(c) 2016 Elsevier B.V. All rights reserved.

\section{Introduction}

Alexithymia (literally meaning "no words for feelings") was first introduced by Sifneos (1973) to describe psychosomatic patients characterized by difficulties in identifying and communicating personal feelings, constriction of emotional expressions and fantasies, and a thinking style preoccupied by external events and limited introspections (Bankier et al., 2001; Nemiah et al.,

\footnotetext{
* Corresponding author at: Laboratory of Neuropsychology, The University of Hong Kong, Pokfulam Road, Hong Kong.
}

1976; Sifneos, 1973). Contemporary studies have identified alexithymia as a personality trait (Salminen et al., 2006), with prevalence estimated at $\sim 10 \%$ amongst the general population (Franz et al., 2008; Mattila et al., 2006), and a predisposing factor for psychopathologies (Bankier et al., 2001; Taylor and Bagby, 2004).

1.1. Relationship between alexithymia and Major Depressive Disorder (MDD)

In particular, alexithymia and MDD are strongly related. Not only is there a much higher prevalence of alexithymia reported amongst the MDD population ( 23-46\%) (Honkalampi et al., 
2000; Saarijärvi et al., 2001), but also people with alexithymia were found to have aggravated depressive symptoms, especially the somatic/affective symptoms of MDD (Guidi et al., 2011; Honkalampi et al., 1999). Such correlations allow alexithymia to be proposed as a potential candidate for subtyping MDD (Vanheule et al., 2007). Nevertheless, inconsistent findings have also been reported. A recent prospective study had found that alexithymia cannot be used to predict MDD, as based on the general population in Finland (Honkalampi et al., 2010). Factor analyses had also found that although alexithymia and depression were separate constructs (Marchesi et al., 2000; Parker et al., 1991), there were frequent overlaps during the measures conducted for both conditions (Hintikka et al., 2001). Hence, the relationship between alexithymia and MDD is relatively unclear.

\subsection{Behavioral characteristics of alexithymia in MDD and nonclinical samples}

Furthermore, alexithymia is a multi-dimensional construct with both cognitive and affective characteristics (Bermond et al., 2007; Taylor and Bagby, 2004). In fact, by reviewing past behavioral studies of alexithymia, distinct characteristics of alexithymia had been reported, depending on whether MDD or nonclinical samples were recruited. Studies taken from nonclinical samples had found that people with alexithymia have reduced sensitivity regarding response to emotional stimuli (Lane et al., 1996; Parker et al., 1993), attenuated interoceptive awareness and physiological reactivity that reflect diminished emotional experience (Herbert et al., 2011). This group has also had tendencies to focus on somatic sensations rather than symbolic representations of words and images upon emotional arousal (Karlsson et al., 2008). In contrast, studies of MDD patients had reported that alexithymia was associated with heightened emotional and physiological arousals, which were interpreted as displacement behaviors for compensating their difficulties in cognitive regulation of emotional experiences (Troisi et al., 2000). Another postulation also suggested that symptoms of helplessness and hopelessness among MDD patients with alexithymia were associated with their attribution of emotional experiences to situational, dispositional or psychological causes due to difficulties in identifying and expressing bodily sensations and feelings (Duddu et al., 2003). As explained by a recent review-study: when capacities regarding both the experiencing and cognitive processing of emotional information are reduced (affective characteristics of alexithymia), the need for emotional regulation was relatively low (drawn from nonclinical samples); whereas for the case where the capacities regarding experiencing emotional information were high but capacities regarding cognitive processing of the information were low (cognitive characteristics of alexithymia), reduced cognitive regulation would lead to experiences of emotions being intensified (especially negative ones, as in MDD patients) (van der Velde et al., 2013). Nevertheless, empirical evidence is required to corroborate such postulations.

\subsection{Major hypotheses on the neural correlates for the cognitive characteristics of alexithymia}

There is not yet a universally accepted theory on the neural correlates of alexithymia and various brain regions have been hypothesized for their roles in shaping the cognitive and affective characteristics of alexithymia.

One prominent view holds that damages at the corpus-callosum (CC) would lead to the cognitive characteristics of alexithymia due to the compromised interhemispheric transferral of information, which would interfere the coordinations of the two cerebral hemispheres in due course of cognitive processing of emotional information (Larsen et al., 2003). The CC hypothesis was originally proposed in study of split-brain patients (Hoppe and Bogen, 1977) and more recent studies also found supporting evidence (Lumley and Sielky, 2000; Parker et al., 1999; Romei et al., 2008). Nevertheless, direct neuroimaging evidence is lacking, while questions have been raised on the nature and directionality of the proposed impairments during cognitive transferral of information (Tabibnia and Zaidel, 2005).

\subsection{Major hypotheses on the neural correlates for the affective characteristics of alexithymia}

Hypotheses on the neural correlates for the affective characteristics of alexithymia are more diverse. A longstanding view proposed deficits at the right hemisphere (or preference of the left hemisphere) (Bermond et al., 2005; Paradiso et al., 2011) would lead to dysfunctional perception and regulation of emotional behaviors (Borod et al., 1998). More recent evidence have focused on the role of the anterior cingulate cortex (ACC), although findings were mixed. Some studies found activities at ACC correlated negatively with alexithymia and suggested reductions in attention and response selection (Chester et al., 2015; Kano et al., 2003; Lane et al., 1998). Other studies claimed that alexithymia was correlated with increased activity at the dorsal ACC and implied this as the defense for increasing emotional inhibition (Pouga et al., 2010). Mixed findings were similarly reported regarding correlations between alexithymia and gray-matter measures of ACC in structural studies, with positive (Gündel et al., 2004), negative (Borsci et al., 2009; Ihme et al., 2013) and non-significant (Heinzel et al., 2012) results all being reported. To explain these controversial findings, an inverted U-shaped activation pattern has been proposed for the dorsal ACC, meaning that when tasks are low in cognitive load, ACC activities would increase with alexithymia, while when cognitive load in the tasks becomes strenuous, ACC activities associated with alexithymia would decrease (McRae et al., 2008; van der Velde et al., 2013). Another possible explanation for the differences in these findings might be the still debating functions of the dorsal ACC (which may be related with cognitive regulation, or appraisal and expression of emotions) (Etkin et al., 2011). Nevertheless, neuroimaging studies consistently found that dorsal ACC is coupled with activities in the right anterior insular (Paulus et al., 2003; Paulus and Stein, 2006), a brain region proposed for somatotopic representation of integrated sympathetic (versus left for parasympathetic) information from posterior-to-anterior progression (Craig, 2009). Hence, we speculate that the ACC and right hemisphere hypotheses for the affective characteristics of alexithymia may be linked with this strong coupling between ACC and the right-lateralized insular for processing of afferent information. Corroborating evidence can be found in the observation that reduced ability in labeling and identifying emotional faces in healthy subjects with alexithymia, particularly when they were under perceptual or temporal constraints, was accompanied by the reduced activations of the limbic and paralimbic brain regions (including the insular) (Ihme et al., 2014; Reker et al., 2010). In sum, a large body of research has suggested that dorsal ACC and right-lateralized deficits are related to alexithymia, particularly the affective characteristics associated with perception and experience of emotions, yet the exact underpinning mechanisms are still largely unknown.

A possible limitation in the existing body of literature is that most of the studies are based on gray-matters, while the role of white-matter fibers for providing connections of ACC with insular and other related brain regions might have been overlooked. To fill this gap, it would be important to identify the white-matter correlates for the affective characteristics of alexithymia. We hypothesized that disruptions at the right superior longitudinal 
fasciculus (SLF), a longitudinal tract proposed for posterior-toanterior (parietal-frontal) connection, may affect emotional attention for controlling perception and experience of emotions (Pourtois et al., 2013), as right-lateralization of this tract has also been found to be associated with the control of visual spatial attention (De Schotten et al., 2011). This proposal is supported by a recent voxel-based morphometry (VBM) study examining alexithymia in nonclinical subjects, which reported that affective characteristics of alexithymia were associated with both lower white-matter volumes of the right SLF and lower gray-matter volumes of the medial-orbitofrontal-cortex (while cognitive characteristics was associated with lower gray-matter volume of the ACC) (van der Velde et al., 2014).

\subsection{The current study}

White-matter connectivity has been demonstrated as a significant index for diagnosing and tracking brain dysfunctions in people with psychiatric disorders (Lim and Helpern, 2002). However, direct relationship between white-matter connectivity and alexithymia are rarely examined. Only a recent study on schizophrenia reported that alexithymia was negatively correlated with white-matter integrity at the CC, the left SLF, the inferior longitudinal fasciculus (ILF) and several other tracts (Kubota et al., 2012). Therefore, in the current study, we examined the whitematter connectivity of alexithymia with diffusion tensor imaging (DTI) data (Basser and Pierpaoli, 1996). Specifically, we explored the relationship of alexithymia and depression by studying the white-matter connectivity (indexed by fractional anisotrophy, FA) in a group of patients diagnosed with first-episode MDD and a matched group of nonclinical controls. Based on the above discussions about the existing evidence of the neural correlates of cognitive and affective characteristics of alexithymia (see Sections 1.3 and 1.4), we hypothesized CC and right SLF as the white-matter correlates for cognitive and affective characteristics of alexithymia respectively. Moreover, following the above discussions on the behavioral characteristics of alexithymia (see Section 1.2) and the speculations by van der Velde et al. (2013), we proposed that MDD and nonclinical groups would be associated with cognitive and affective characteristics of alexithymia respectively. Combining both, we predicted that there would be significant group interaction effect for the white-matter correlates of alexithymia, with MDD group at CC (hypothesized white-matter correlates of cognitive characteristics) and nonclinical group at right SLF (hypothesized white-matter correlates of affective characteristics).

Analysis would be based on tract-based spatial statistics (TBSS), a technique recommended for reducing risks of local misalignments in VBM (Smith et al., 2006, 2007) and has been adopted in various previous studies of MDDs (Guo et al., 2012a, 2012b).

Moreover, since white-matter tracts were responsible for providing integral routes of communication for anatomically separated brain areas, connectivity disturbance of these tracts might induce extended impacts on the functional connectivity (temporal correlations of signal fluctuations) of spatially separated neural networks (Greicius et al., 2009). Therefore, seed-based restingstate functional connectivity magnetic resonance imaging (RfcMRI) analyses (Fox and Raichle, 2007) were conducted to locate the remote brain regions with disturbances in functional coordination associated with alexithymia. As networks identified by both resting-state and task-related techniques were highly corresponded (Smith et al., 2009), resting-state method was adopted to avoid biases from the difference in individual task performance (Fox and Raichle, 2007).

\section{Methods}

\subsection{Subjects and behavioral measures}

The current study involved only females as test subjects, to avoid the influence of gender effect on the results (Levant et al., 2009). One group of the participants included 22 outpatients (MDD group, ages ranged from 25 to 55 years) receiving treatment at the David Trench Rehabilitation Center upon diagnosis of their first episode of MDD but not any other affective disorder according to the criteria in DSM-IV (American Psychiatric Association, 1994). Although we attempted to minimize the effect of medication by recruiting only newly diagnosed patients, these patients were taking anti-depressants at the time of the experiment (ranged from 3 to 16 weeks). They also fulfilled the following inclusion criteria: Chinese, right-handed, healthy individuals physically without histories of organic brain disorders, traumatic brain injuries, substance abuse/dependence disorders, psychotic disorders, and any other formal diagnosis of anxiety disorders. The other group was 21 nonclinical controls (NC group, ages ranged from 25 to 64 years) recruited from the local community. They had no history of psychological disorders and were matched with the MDD group by age, education level and estimated intelligence as assessed by the Raven's Progressive Matrices (Raven, 2000). All participants were assessed on the degree of alexithymia by the Chinese version of the 20-item Toronto Alexithymia Scale (TAS-20C, Bagby et al., 1994; Zhu et al., 2007) and the level of depression was assessed by the Chinese version of the Beck Depressive Inventory (BDI-II-C, Byrne et al., 2004). They had all given their written and informed consent, as approved by the Institutional Review Board of the University of Hong Kong/Hospital Authority Hong Kong West Cluster. Table 1 shows the demographic variables and affective profiles for the two groups.

Consistent with existing data, significant correlations between the rate of alexithymia (TAS-20-C) and degree of depression (BDIII-C) were found, both across groups $(r=.69, p<.001)$ and for each group separately (MDD group: $r=.546, p<.05$; NC group: $r=.701$, $p<.001)$.

\subsection{Data acquisition and preprocessing}

DTI data were acquired from a 3.0 T Philips scanner equipped with a standard 8-channel head coil, using 32 isotropically

\section{Table 1}

Demographic characteristics and affective profiles of MDD patients and nonclinical controls, including the group means, SDs, values of independent samples $t$-tests, and associated $p$-values.

\begin{tabular}{lllll}
\hline & $\begin{array}{l}\text { MDD group } \\
(n=22) \\
\text { mean }(S D)\end{array}$ & $\begin{array}{l}\text { NC group } \\
(n=21) \\
\text { mean (SD) }\end{array}$ & t-value & $p$-value \\
& & & & \\
\hline $\begin{array}{l}\text { Demographics } \\
\text { Age }\end{array}$ & $37.6(8.32)$ & $41.9(11.04)$ & -1.4201 & .163 \\
Years of education & $12.9(2.83)$ & $13.4(4.55)$ & -.4102 & .684 \\
RPM & $247.5(10.51)$ & $252.2(12.80)$ & -1.3101 & .198 \\
Length of medication & $6.81(3.26)$ & NA & NA & NA
\end{tabular}

(weeks)

Affective profiles

BDI-II-C

TAS-20-C

25.9 (13.35)

59.1 (11.65)

7.3 (7.17)

49.8 (10.28)

5.7332

$<.001^{* * * *}$ $.009^{* * *}$

Note: $\mathrm{MDD}=$ major depressive disorder, $\mathrm{NC}=$ nonclinical control, $\mathrm{RPM}=$ raw score of the Raven's Standard Progressive Matrices Test, BDI-II-C=Chinese Beck Depression Inventory-II, TAS-20-C=20-Item Toronto Alexithymia Scale (Chinese version).

$* * p<.01$.

**** $p<.001$. 
distributed orientations for diffusion-sensitizing gradients at a $b$ value of $1000 \mathrm{~s} / \mathrm{mm}^{2}$, with the scanning parameters as follows: time of echo $(\mathrm{TE})=65 \mathrm{~ms}$, repetition time $(\mathrm{TR})=11,375 \mathrm{~ms}$, flip angle $=90^{\circ}$, field of view $(\mathrm{FOV})=225 \mathrm{~mm}$, acquisition matrix $=112 \times 112$, slice thickness $=2 \mathrm{~mm}$, slice gap $=0 \mathrm{~mm}, 74$ slices in axial plane, voxel size $=2 \times 2 \times 2 \mathrm{~mm}^{3}$, and total acquisition time $=830 \mathrm{~s}$.

The collected DTI data were then processed using the FMRIB Software Library version 5.0.2 (FSL, Smith et al., 2004). All source data were corrected for head motion and eddy currents by affine registration referencing the first $b=0$ image, using the FMRIB Diffusion Toolbox (FDT, part of FSL). FA images were subsequently generated from the corrected data by fitting diffusion tensors with the DTIFIT (part of FSL) and by extractions made from the brain using the mask generated by the Brain Extraction Tool (BET, part of FSL, Smith, 2002). Tract-Based-Spatial Statistics version 1.2 (TBSS, part of FSL) was engaged to create a mean FA skeleton for representing the centers of all tracts that are common to all participants, by thinning and applying a threshold of .2 on the FA value on the mean FA image after it was normalized (nonlinear registration to the $1 \times 1 \times 1 \mathrm{~mm}^{3}$ Montreal Neurological Institute 152 space). The mean FA skeleton would then be applied to individual FA images to restrict the evaluation of diffusion parameters prior to projection to individual skeletonized FA images. These skeletonized FA images were then applied and used for analysis by voxel-wise permutation-based nonparametric inference using the FSL Randomize version 2.5 (Nichols and Holmes, 2002) using 5000 permutations with the threshold-free cluster enhancement option (TFCE).

R-fcMRI data were acquired with a $T 2 *$-weighted echo-planar imaging sequence for 6 min (during which the participants were awake but had their eyes closed) inside the same MRI machine as the DTI data, with the scanning parameters as follows: time of echo $(\mathrm{TE})=30 \mathrm{~ms}$, repetition time $(\mathrm{TR})=2000 \mathrm{~ms}$, flip angle $=90^{\circ}$, field of view $(F O V)=230 \mathrm{~mm}$, acquisition matrix $=64 \times 64$, slices thickness $=3.5 \mathrm{~mm}$, slice gap $=0 \mathrm{~mm}, 40$ slices in axial plane, voxel size $=3.5 \times 3.5 \times 3.5 \mathrm{~mm}^{3}$. Preprocessing of the acquired functional images and single-subject level calculation of functional connectivity were performed using DPARSF (Yan and Zang, 2010) and REST (Song et al., 2011), while group-level analysis was conducted by Statistical Parametric Mapping (SPM8; Wellcome Department of Cognitive Neurology, London, UK), all running on a commercial software package (MATLAB, v7.14, The MathWorks Inc., Natick, MA, 2000).
The first 10 slices of all source R-fcMRI data were discarded before the preserved data were spatially realigned to the middle slice in order to adjust for the differences in the time of acquisition of multiple slices within an image and corrected for head-motion. The resulting images were then co-registered to their individual high-resolution T1 image equivalents, and then normalized to the $3 \times 3 \times 3 \mathrm{~mm}^{3}$ MNI template by unified segmentation and then spatially smoothed with a $6 \mathrm{~mm}$, full-width, half-maximum (FWHM) Gaussian kernel. Nuisance variables were regressed out, including the six head motion parameters, mean signals of the whole brain (the global trend), white-matter and cerebrospinal fluid. These images, after removal of linear trends and after being band-pass filtered (at .01-.08 Hz) to remove the cardiac and respiratory signals, were ready for statistical analysis.

\subsection{Data analysis}

Age was controlled in all of the following analyses to remove any discrepancies or impacts on the results due to age-related factors, as their effects had been observed continually in studies of depression (Mirowsky and Ross, 1992) and alexithymia (Mattila et al., 2006). Thus, it would be undesirable for this effect to be restated in the present study.

For DTI data, firstly, in order to test the hypothesis that alexithymia would be associated with white-matter integrity at different locations for the two groups, voxel-wise multiple regression in separate analyses, using CC and right SFL as region of interest (ROI) based on a priori hypotheses, were conducted on the skeletonized FA images across all participants, with group and alexithymia (measured by TAS20-C scores, demeaned before entered into the model) as regressors. Any significant group interaction effect would be further examined by extracting the mean FA values from the result clusters and entered into linear regression models for analyses.

Secondly, to test that CC and right SLF are white-matter correlates of alexithymia for MDD and NC group respectively, voxelwise multiple regression analyses, using these tracts as ROI, were conducted on each group separately, with any potential effect of depression removed by entering the BDI-II-C scores as covariate of no interest. Mean values of FA and other apparent diffusion coefficients (ADCs), including mean diffusivity (MD, volumetric measure for the degree of diffusion), radial diffusivity (RD, diffusivity perpendicular to the principal axons) and axial diffusivity (AD, diffusivity parallel to the principal axons), would also be extracted

Table 2

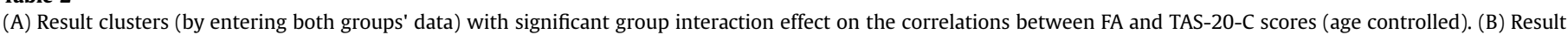
clusters (by entering data in each group separately) with significant correlations between FA and TAS-20-C scores (age, BDI-II-C scores controlled)

\begin{tabular}{|c|c|c|c|c|c|c|c|c|c|c|}
\hline ROI & $\mathrm{CC}$ & \multirow{3}{*}{$p$-value } & \multirow[b]{3}{*}{$\mathrm{x}$} & \multirow{3}{*}{$\begin{array}{l}\text { Right } \\
\text { SLF } \\
\text { Peak } \\
\text { voxel } \\
\mathrm{y}\end{array}$} & \multirow[b]{3}{*}{$\mathrm{z}$} & \multirow{3}{*}{$\begin{array}{l}\text { No. of voxels } \\
1 \times 1 \times 1 \mathrm{~mm}^{3}\end{array}$} & \multirow{3}{*}{$p$-value } & \multirow[b]{3}{*}{$\mathrm{x}$} & \multirow{3}{*}{$\begin{array}{l}\text { Peak } \\
\text { voxel } \\
\mathrm{y}\end{array}$} & \multirow[b]{3}{*}{$\mathrm{z}$} \\
\hline \multirow[t]{2}{*}{ Clusters } & No. of voxels & & & & & & & & & \\
\hline & $1 \times 1 \times 1 \mathrm{~mm}^{3}$ & & & & & & & & & \\
\hline \multicolumn{11}{|c|}{ (A) Clusters with significant group interaction effect (age controlled) } \\
\hline 1 & 2198 & $.005^{* * *}$ & -8 & 0 & 28 & 221 & $.022 *$ & 45 & -6 & 26 \\
\hline 2 & 49 & $.046^{*}$ & -7 & 29 & 1 & 77 & $.031^{*}$ & 31 & 4 & 30 \\
\hline 3 & 40 & $.047^{*}$ & -8 & -35 & 23 & & & & & \\
\hline 4 & 23 & $.047^{*}$ & -10 & -44 & 17 & & & & & \\
\hline 5 & 13 & $.049 *$ & -13 & 30 & -8 & & & & & \\
\hline \multicolumn{11}{|c|}{ (B) Clusters with significant correlations with in any one group (age, BDI-II-C scores controlled) } \\
\hline & \multicolumn{5}{|c|}{ CC (positive correlation, MDD group) } & \multicolumn{5}{|c|}{ Right SLF (negative correlation, NC group) } \\
\hline 1 & 199 & $.029^{*}$ & 5 & -12 & 26 & 356 & $.012^{*}$ & 47 & -6 & 25 \\
\hline 2 & 33 & $.046^{*}$ & -13 & -18 & 31 & 16 & $.046^{*}$ & 31 & 2 & 30 \\
\hline
\end{tabular}

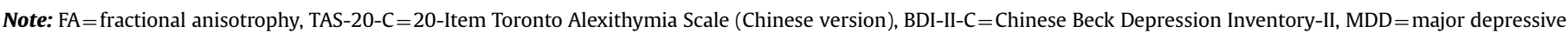
disorder, $\mathrm{NC}=$ nonclinical control.

$* p<.05$

$* * p<.01$ (FWE-corrected). 


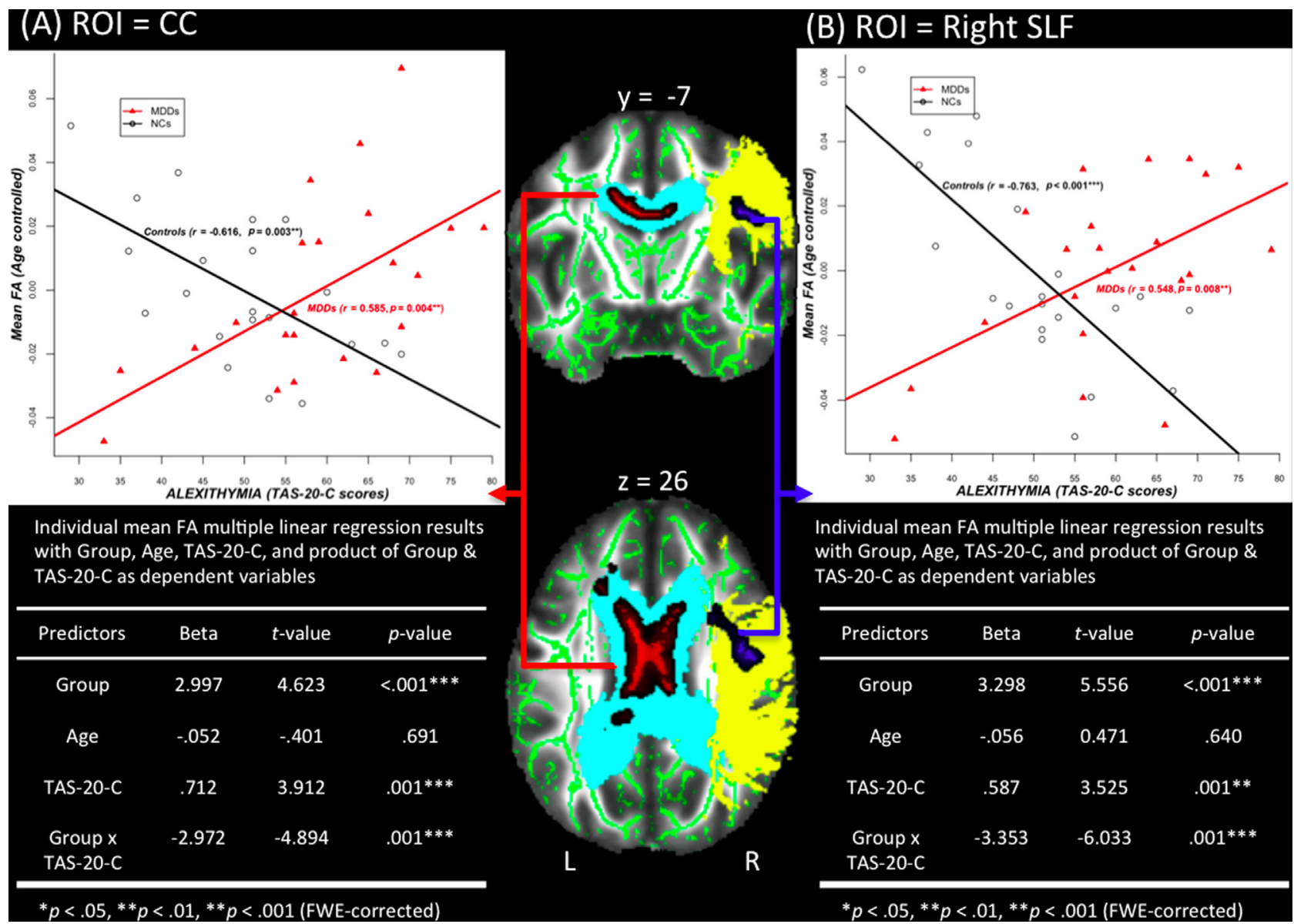

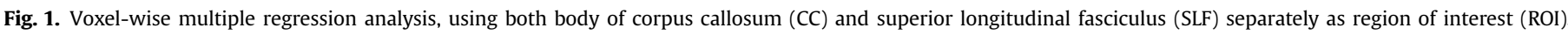

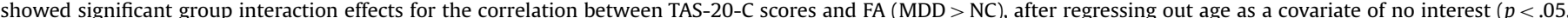

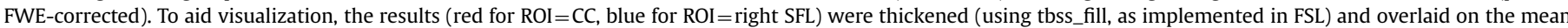

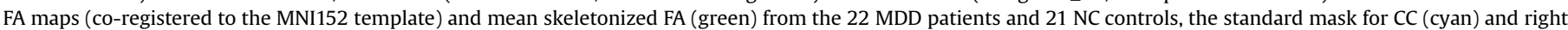

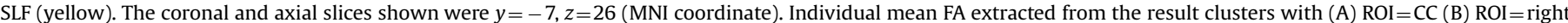

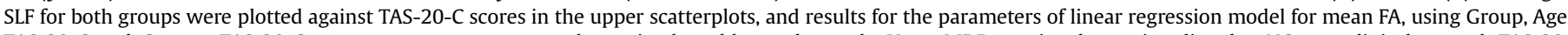

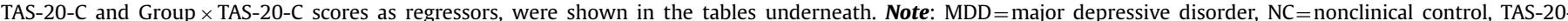

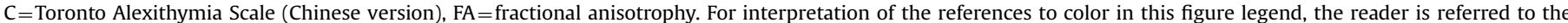
web version of this article.

from any significant result cluster for further inference of the FA results.

Statistical threshold for DTI analysis was set at $p<.05$, controlled for family-wise error rate (FWE-corrected). White-matter tracts obtained in the result clusters were identified with reference to the two white-matter atlases-the ICBM-DTI-81 white-matter labels atlas and the JHU white-matter tractography atlas-provided by Johns Hopkins University (http://cmrm.med.jhmi.edu) (Mori et al., 2005).

For r-fcMRI data, separate analyses were conducted for each group, based on the result clusters identified in the DTI analysis within the ROIs of the a priori hypotheses (that could be fortunately be confirmed) for the respective group. Specifically, by adopting the method for geometrical segmentation of the midsagittal CC (Hofer and Frahm, 2006) and SFL (Makris et al., 2005), we identified the specific segments within CC and right SFL where the white-matter correlates were located. Termination points were determined as the brain regions (ROIs) that are connected by these specific tract segments. We then engage these ROIs as seeds for functional connectivity calculation, by having the mean neural activities of all voxels within the corresponding seed ROIs for each participant extracted and correlated (using Pearson's correlation, $r$ ) with all other brain voxels for every time point within the time course of the preprocessed r-fcMRI data. These were then transformed to Fisher's $Z$ scores to produce the standardized functional connectivity ( $\mathrm{z}-\mathrm{fc}$ ) maps. The $\mathrm{z}-\mathrm{fc}$ maps for participants in each group were entered into second-level, voxel-wise, multiple regression models for identifying the locations where significant correlations between $\mathrm{z}-\mathrm{fc}$ and TAS-20-C scores would be found, after controlling BDI-II-C scores. Results were voxel-level height thresholded at $p<.001$ and peak- or cluster-level $p<.05$ (FWEcorrected). Brain anatomical regions were identified with reference to Anatomical Automatic Labeling (AAL, Tzourio-Mazoyer et al., 2002).

\section{Results}

\subsection{Structural connectivity analysis}

3.1.1. Comparison on the correlations of FA and alexithymia across MDD and NC groups using CC and right SLF as ROI

Multiple clusters where observed with significant group interaction effect on the correlations between TAS-20-C scores and FA (only MDD $>$ NC, not MDD $<$ NC), by adopting the body of CC and the right SLF separately as ROIs (see Table 2A). No cluster was found to have significant correlation of FA, either by group or TAS20-C scores alone. 


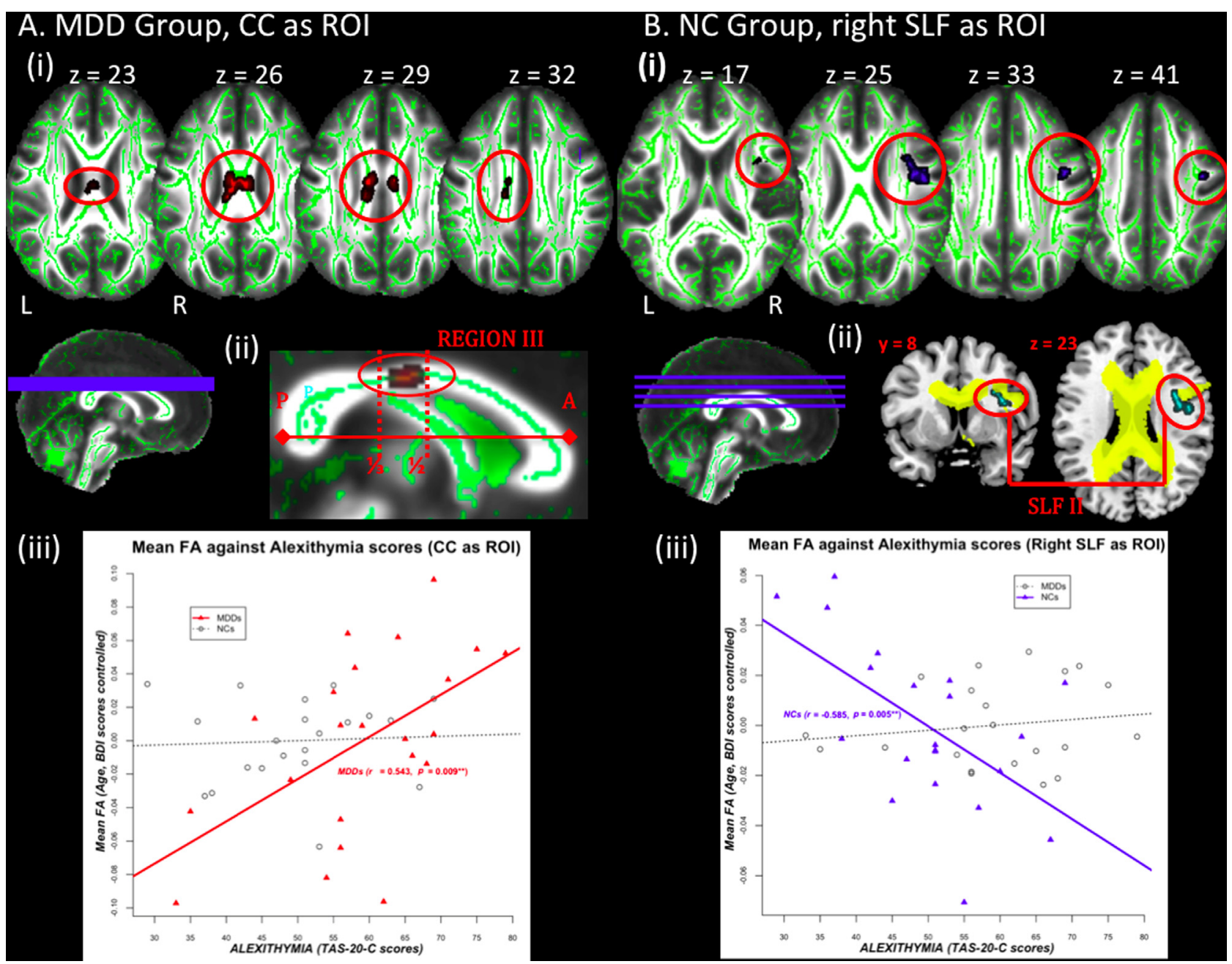

Fig. 2. (A) (i) Voxel-wise multiple regression analysis using $\mathrm{CC}$ as ROI showed significant positive correlations between TAS-20-C scores and FA in the MDD group after regressing out both age and BDI-II-C as covariates of no interest $(p<.05$, FWE-corrected). To aid visualization, the results (red) were thickened (using tbss_fill, as implemented in FSL) and overlaid on the mean FA maps (co-registered to the MNI152 template) and mean skeletonized FA (green). The axial slices shown were from $z=23$ to 32 (MNI coordinate). (ii) The result clusters overlaid on the mid-sagittal view of the mean FA map showed that it mainly lied on region III of the corpus callosum (posterior half minus posterior third) based on the mid-sagittal corpus callosum topology (Hofer and Frahm, 2006). (iii) Scatterplots of mean FA extracted from the result cluster (from 2(A) (i)) showed that only mean FA from the MDD group was significantly correlated with TAS-20-C scores, after controlling for age and BDI-II-C scores (red solid line indicating positive correlation), but not those from the NC group. (B) (i) Voxel-wise multiple regression analysis using right SFL as ROI showed significant negative correlations between TAS-20-C scores and FA in the NC group after regressing out both age and BDI-II-C as covariates of no interest ( $p<.05$, FWE-corrected). To aid visualization, the results (blue) were thickened (using tbss_fill, as implemented in FSL) and overlaid on the mean FA maps (co-registered to the MNI152 template) and mean skeletonized FA (green). The axial slices shown were from $z=17$ to 41 (MNI coordinate). (ii) The result clusters overlaid on the coronal and axial view of a standard T1-weighted brain template, with the nearby brain regions (yellow) to indicate that it mainly lied on subcomponent II SLF (Makris et al., 2005). (iii) Scatterplots of mean FA extracted from the result cluster (from (B) (i)) showed that only mean FA from the NC group was significantly correlated with TAS-20-C scores, after controlling for age and BDI-II-C scores (blue solid line indicating negative correlation), but not those from the MDD group. Note: $\mathrm{MDD}=$ major depressive disorder, $\mathrm{NC}=$ nonclinical control, $\mathrm{CC}=\mathrm{Corpus}$ Callosum, $\mathrm{SLF}=$ superior longitudinal fasciculus, $\mathrm{ROI}=$ region of interest, TAS-20-C=Toronto Alexithymia Scale (Chinese version), BDI-II-C $=$ Chinese Beck Depression Inventory-II, $\mathrm{FA}=$ fractional anisotrophy. For interpretation of the references to color in this figure legend, the reader is referred to the web version of this article.

To further examine this interaction effect of group, mean FAs for individual participants were extracted from the result clusters and plotted against TAS-20-C score, as well as entered into linear regression model. The results showed that all three regressors, including group, TAS-20-C score and their cross product, were significant predictors of mean FA (see Fig. 1).

The two overall models were also significant (CC as ROI: $F$ $(4,38)=6.612, p<.001, R^{2}=.410$; right SLF as ROI: $F(4,38)=9.726$, $\left.p<.001, R^{2}=.506\right)$. Mean FAs extracted by adopting CC as ROI could be predicted by the following formulas:

MDD group: $F A=.6246-.0001392 * A g e+.001583^{*}$ TAS

NC group: $F A=.7806-.0001392^{*}$ Age $-.001407^{*}$ TAS

Mean FAs extracted by adopting right SLF as ROI could be predicted by

MDD group: $\mathrm{FA}=.4077+.0001669^{*} \mathrm{Age}+.001454^{*} \mathrm{TAS}$

NC group: $F A=.5988+.0001669^{*}$ Age $-.002302^{*}$ TAS

\subsubsection{Separate group analyses using CC and right SLF as ROI}

As shown in Table 2B, two clusters were found to have significant correlations between FA and TAS-20-C, positively (not negatively) for MDD group with CC as ROI, and negatively (not positively) for NC group with right SLF as ROI, both controlled for BDI-II-C scores.

Fig. 2A shows the clusters within CC where significant positive correlations between FA and TAS-20-C scores were observed.

By method of geometrical segmentation of mid-sagittal CC (Hofer and Frahm, 2006), the result clusters were found to be lying at Region III of CC, that is, the posterior half-minus-posterior third of CC (see Fig. 2A(ii)). As proposed by these authors, this region is comprised of callosal motor fibers (CMFs), which project into the primary motor cortices.

Furthermore, by plotting mean FA extracted from the result clusters against the TAS-20-C scores for each participant, 
Table 3

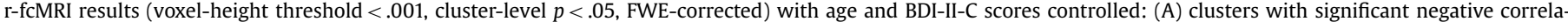

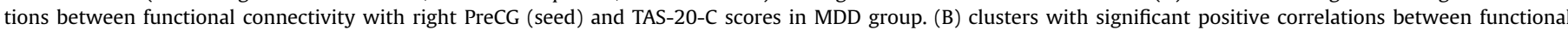
connectivity with right IFG_triang (seed) and TAS-20-C scores and in NC group.

\begin{tabular}{|c|c|c|c|c|c|c|c|c|c|}
\hline \multirow[t]{2}{*}{ Brain Region (BA) } & \multicolumn{3}{|l|}{ Cluster-level } & \multicolumn{3}{|l|}{ Peak-level } & \multicolumn{3}{|c|}{ MNI coordinates } \\
\hline & $p$ (fwe-corr) & $p$ (uncorr) & $k$ & $p$ (fwe-corr) & $p$ (uncorr) & $t$-Value & $x$ & $y$ & $z$ \\
\hline \multicolumn{10}{|c|}{ (A) MDD group (negative correlation) } \\
\hline \multirow{2}{*}{ PCUN/mid-cingulum (BA 23) } & .000 & .000 & 102 & .883 & .000 & 4.99 & 6 & -48 & 36 \\
\hline & & & & .941 & .000 & 4.82 & 6 & -33 & 36 \\
\hline \multirow[t]{3}{*}{ ANG (BA 19/39) } & .010 & .005 & 61 & .986 & .000 & 4.56 & 60 & -60 & 36 \\
\hline & & & & .999 & .000 & 4.28 & 42 & -72 & 42 \\
\hline & & & & 1.000 & .000 & 4.11 & 51 & -66 & 36 \\
\hline \multicolumn{10}{|c|}{ (B) NC group (positive correlation) } \\
\hline SOL (BA 19) & .003 & .000 & 71 & .000 & .000 & 10.18 & 21 & -81 & 27 \\
\hline MOL (BA 19) & & & & 1.000 & .001 & 3.85 & 36 & -81 & 27 \\
\hline
\end{tabular}

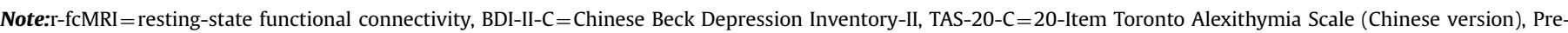

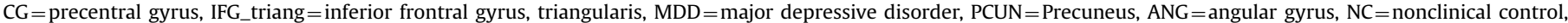
$\mathrm{SOL}=$ superior occipital lobe, $\mathrm{MOL}=$ mid-occipital lobe.

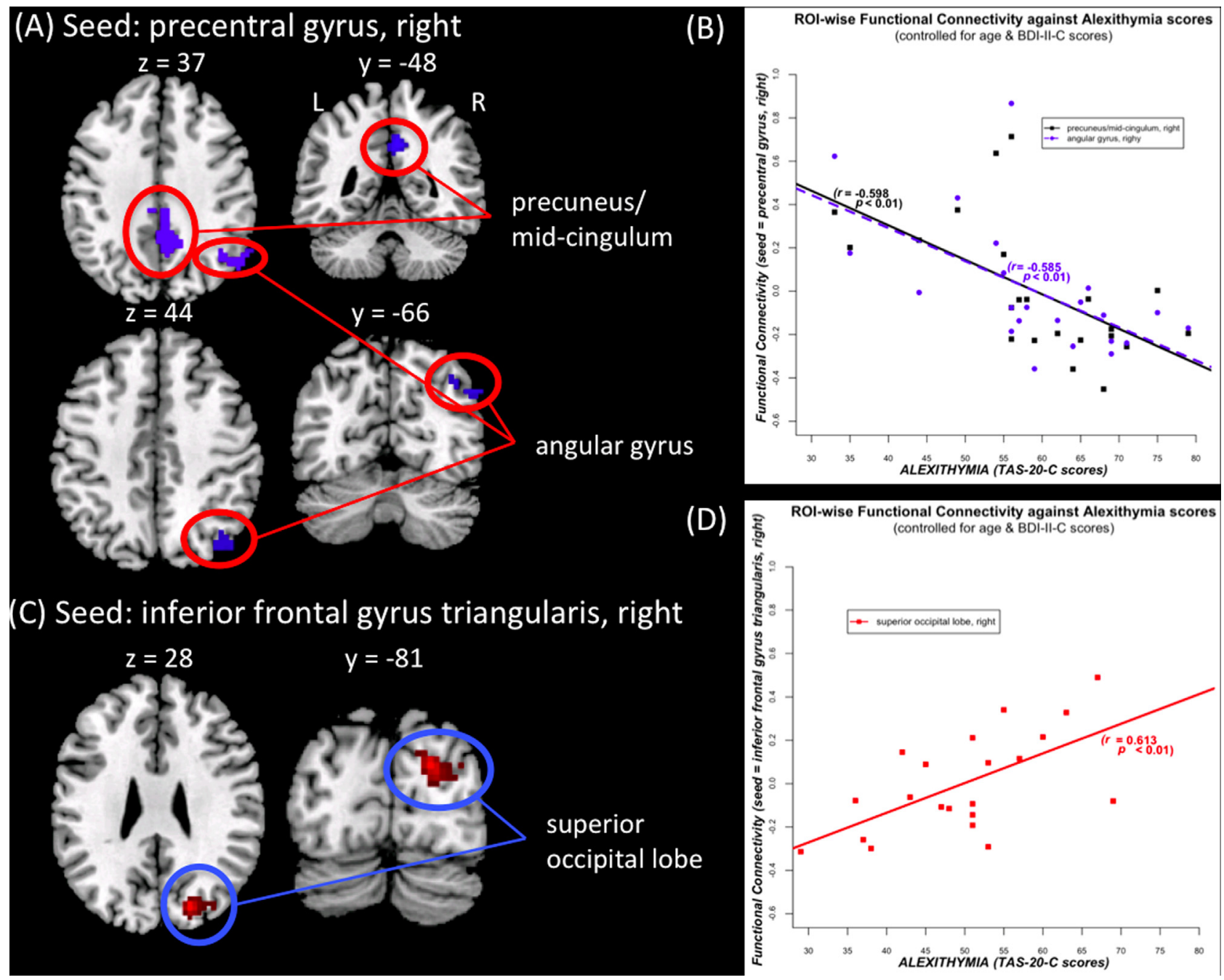

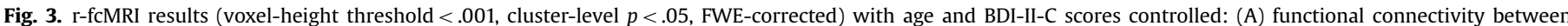

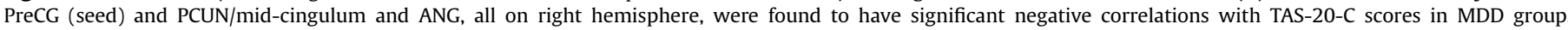

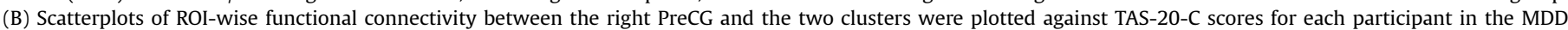

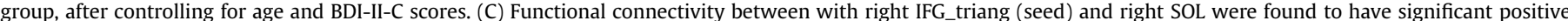

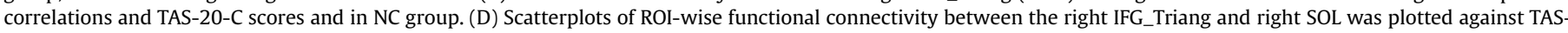

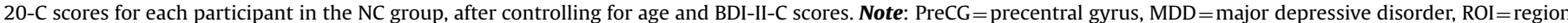

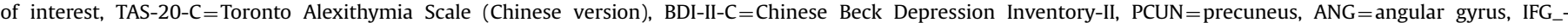
Triang =inferior frontal gyrus, triangularis, $\mathrm{SOL}=$ superior occipital lobe. 
significant positive correlation was only observed in the MDD group $(r=.543, p=.009)$ but not in the NC group $(r=.053, p=.821)$ (see Fig. 2A(iii)). Finally, to investigate the contribution of diffusivity indexes that underpin $\mathrm{FA}$, mean $\mathrm{MD}, \mathrm{RD}, \mathrm{AD}$ were also extracted from the result clusters for individual participants in the MDD group. FA was found to have significant negative correlations with $\mathrm{RD}(r=-.991, p<.001)$ and $\mathrm{MD}(r=-.908, p<.001)$, but not with $\mathrm{AD}(r=.230, p=.395)$. These findings suggested that increasing FA within CC in MDD group was not related to increased diffusivity at the principle axons, but reduced diffusivity as reflected by the reduced volumetric measure (MD) and diffusivity perpendicular to the principal axons (RD). One possible explanation was the reduced diameters of the relatively large and less densely packed CMF axons (Aboitiz et al., 1992; Beaulieu, 2010; Hofer and Frahm, 2006; Paul, 2011).

Fig. 2B presents the clusters within right SLF where significant negative correlations between FA and TAS-20-C scores were found. Similar to the preceding findings, based on the geometrical segmentation of SFL (Makris et al., 2005), the result clusters were found to be located at the subcomponent II of SFL (lateral to the CC but medial to the middle-frontal-gyrus and the pars-opercularis of the inferior-frontal-gyrus) (see Fig. $2 \mathrm{~B}(\mathrm{ii})$ ), which is a major association pathway between the PFC and the posterior parietal region that has been found to be primarily responsible for regulating visual spatial attention for perception (De Schotten et al., 2011).

Again, by plotting mean FA extracted from the result clusters with regard to each participant against the TAS-20-C scores, significant negative correlations were observed in the NC group $(r=-.585, p=.005)$ but not in the MDD group $(r=.150, p=.504)$ (see Fig. 2B(iii)). Examination of correlations between mean FA and mean $M D, R D, A D$ extracted from the result clusters for individual participant found significant positive correlation for $\mathrm{AD}$ $(r=.665, p=.003)$ and significant negative correlation for $\mathrm{RD}$ $(r=-843, p<.001)$, but only marginal significant correlation for MD $(r=-.458, p=.073)$. These further affirmed that the decreasing FA in association with TAS-20-C scores at the right SLF for the NC group was related to decreasing white-matter diffusivity.

\subsection{Resting-state functional connectivity analysis}

\subsubsection{Seed-based analysis for MDD group}

Functional connectivity was computed based on seeds at bilateral-precentral-gyri (PreCG, AAL label of primary motor cortex), the termination points connected by CMFs (neural correlate of alexithymia in MDD group revealed in structural connectivity analysis). Significant negative correlations with TAS-20-C scores were found for the functional connectivity between the right PreCG and clusters at the right precuneus (PCUN)/mid-cingulum (BA 23) and right angular gyrus (ANG, BA 19/39) (see Table 3A and Fig. 3A). Scatterplot showing the correlations between TAS-20-C scores and the ROI-wise functional connectivity (between right PreCG and the results clusters) with regard to each participant in the group is presented in Fig. 3B. No significant positive correlation between TAS-20-C and functional connectivity with the right PreCG and no significant positive or negative correlation between TAS-20-C and functional connectivity with the left PreCG were found.

\subsubsection{Seed-based analysis for NC group}

PFC was the frontal termination point connected by SLF II (neural correlate of alexithymia in NC group revealed in structural connectivity analysis), particularly the right IFG, which is connected to the anterior insular for representing the afferent bodily feelings from the sensory network (Craig, 2009). Thus, functional connectivity was computed using the right IFG (including labels in AAL: IFG-opercular, BA44/48; IFG-triangularis, BA45; IFG-orbital,
BA47) as seeds. Significant positive correlation with TAS-20-C scores was found for the functional connectivity between right IFG-triangularis (IFG_triang, BA45) and the right superior-occipital-lobe (SOL, BA 19) (see Table 3B and Fig. 3C). Scatterplot showing the correlation between TAS-20-C scores and the ROIwise functional connectivity (between right IFG-triang and right $\mathrm{SOL}$ ) extracted for each participant in the group is presented in Fig. 3D.

\section{Discussion}

This study explored the relationship between alexithymia and MDD based on white-matter connectivity and seed-based restingstate functional connectivity. The results confirmed our a priori hypothesis that the alexithymia-correlated white-matter can be located at distinct locations in the CC for the MDD group and in the right SLF in the NC group. Further study of r-fcMRI analyses, using the seeds derived from white-matter connectivity results, revealed that there are extended impacts of the microstructural changes associated with alexithymia on remote functional networks. These findings will be discussed in more details in the following section in light of the distinct behavioral characteristics displayed by people with alexithymia among the two groups (discussed in Section 1.2). To the best knowledge of the authors, this is the first combined structural (DTI) and functional (r-fcMRI) connectivity study for examining the relationship between alexithymia and MDD.

\subsection{Structural connectivity of alexithymia in MDD and NC groups}

DTI analysis showed that alexithymia was associated with reduced connectivity at the CC in the MDD group, particularly at the CMFs that specialized in the transfer of sensorimotor information. Our findings provided supporting neural evidence for the hypothesis regarding the CC (Larsen et al., 2003) and the association of MDD with cognitive characteristics of alexithymia (van der Velde et al., 2013). They were also consistent with previous findings that decreasing white-matter connectivity in the CC was associated with increasing degree of alexithymia in people diagnosed with schizophrenia (Kubota et al., 2012), and also increasing severity of depression (positively correlated with alexithymia) in MDD patients (Cole et al., 2012).

In contrast, DTI results in the NC group indicated that alexithymia was associated with reduced diffusivity at the right SLF, particularly at the subcomponent responsible for connecting the PFC with posterior region responsible for regulating of spatial attention during visual perception. The results corroborated previous findings regarding the right SLF as the neural correlate of affective characteristics of alexithymia in healthy subjects (van der Velde et al., 2014).

\subsection{Functional connectivity of alexithymia associated with the structural changes in MDD and NC groups}

For the MDD group, only functional connectivity in the right, but not the left, hemisphere was affected, which may suggest the impairment of CC for the left-to-right direction. This concurred with the postulation that the right hemisphere is the dominant center for processing emotional information and regulation of emotional feelings incurred subjectively (Bermond et al., 2005; Paradiso et al., 2011). In particular, alexithymia in the MDD group was found to be associated with reduced connectivity among the right PreCG and two right brain regions in the DMN, namely the PCUN/mid-cingulum and ANG (Buckner et al., 2005; Uddin et al., 2009). These were essential regions involved in processing of self- 
related attention and memories of emotional information (Greicius et al., 2009; Kober et al., 2008; Seghier, 2013), as well as processing of stimulus value (Lin et al., 2012) and regulation of emotions (Kohn et al., 2014). Similar findings with lower connectivity in the cognitive emotion processing subnetwork of the DMF has also been found in another resting-state study of alexithymia, and the authors interpreted this as associated with the less introspective thinking of alexithymia (Liemburg et al., 2012). For the current study, since reducing diffusivity in association with alexithymia has been observed at the tract connecting the left and right PreCG, particularly at CMF, we proposed to relate the reducing functional coupling with the reducing interhemispheric transfer of information (the CC hypothesis), particularly sensorimotor information, for emotional processing in the right hemisphere. As DMN is associated with spontaneous, rather than goaldirected brain activities (Fox and Raichle, 2007), the reducing functional coupling between the PreCG and emotional processing network in the DMN might reflect reducing automatic evaluation and regulation of emotional information associated with alexithymia. Alternatively, since brain activities are suggested to be switching between the central executive network (CEN) and the DMN (Menon and Uddin, 2010; Sridharan et al., 2008), the reducing functional coupling in DMN would also suggest increasing goal-directed effort (implying greater difficulties) for cognitive evaluation and regulation of emotional information. This increasing effort in cognitive emotional regulation might explain the increasing displacement behaviors, in terms of heightened emotional and physiological arousal (Troisi et al., 2000); as well as the increasing somatic/affective symptoms found in MDD patients with alexithymia (Guidi et al., 2011; Honkalampi et al., 1999).

In contrast, for the NC group, r-fcMRI results showed that alexithymia was associated with increasing connectivity between right IFG_triang and right SOL. This was consistent with the observation in a previous $\mathrm{r}$-fcMRI study that there was higher connectivity at the right IFG for the alexithymic group of a nonclinical sample (Liemburg et al., 2012)and was implied by these authors as reflecting that the alexithymic group engaged suppression techniques, rather than verbalizing strategies, for emotional regulation.

Nevertheless, IFG has been suggested for a role in the selection of context-appropriate attention (Kober et al., 2008), as well as analysis of emotional meaning and affect-labeling (Aron et al., 2004). Specifically, the right IFG_triang has been found to be involved in processing of emotional information and evaluation of affective salience (Rota et al., 2009). In light of the current findings that alexithymia in the $\mathrm{NC}$ group was associated with reduced diffusivity of SLF II, the tract proposed for connecting the IFG and SOL, we suggest that this increasing functional coupling might help to compensate the reducing perceptual information passed from the SOL to IFG_triang for evaluation of emotional salience for exogenous stimuli. This interpretation is consistent with our proposal that affective alexithymia may be associated with disruptions of the highly interconnected network for processing of afferent sensory information, including the ACC, IFG and right anterior insular (Craig, 2009), and also concurred with a previous study which found that joint activation of ACC (as well as the dorsolateral prefrontal-cortex DLPFC and parietal regions) and the anterior insular/IFG was associated with conscious access of visual perceptual information, based on the attention blink paradigm (Kranczioch et al., 2005). Similar evidence was observed in a patient with lesion at the right SOL (BA19), who was found to have both relative lowered level of selective visual spatial attention and high level of affective alexithymia (Ho and Lee, 2013). Another coherent interpretation was offered by the authors of an EEG study, who also suggested that increase in brain reactivity at the anterior and/or posterior areas of the cortex in association with alexithymia (in nonclinical sample), particularly at the right hemisphere and whilst the subjects were watching emotional clips, might reflect increase in their effort for emotional processing (Aftanas and Varlamov, 2007). Moreover, the relationship between capacity to experience emotions and the extent at which emotional significance of environmental stimuli could be identified has long been established in past studies (Lane et al., 1998; Phillips et al., 2003).

In summary, results of the r-fcMRI in NC group were consistent with the characteristic features of people with affective alexithymia, namely tendency to have difficulties in inducing emotional feelings ensuing external stimuli, preferences for diverting attention to external information (Vorst and Bermond, 2001) and mislabeling of somatic sensations (Taylor and Bagby, 2004).

\subsection{Limitations}

Firstly, only the measure for alexithymia, TAS-20-C, was assessed. This mainly measures the cognitive characteristics of alexithymia. Although this enabled comparisons being made with most prior studies, engagement of other alexithymia measures, especially including the affective characteristics, would help validate the current findings. Secondly, only a limited number of female patients were included in the sample due to the difficulties in recruiting patients, especially only patients diagnosed with first episode MDD was recruited to minimize the effect of medication. However, this would limit the generalizability of the current findings and also potentially inflat the effect size of the current findings (Yarkoni and Braver, 2010). Thirdly, due to ethical concerns, the effect of medication was not controlled, as well as the levels of alexithymia across the two groups. Future research would need to better select their samples to minimize these potential confounds.

For the imaging results, the exact microstructural changes represented by the diffusivity indexes had yet to be confirmed. Use of tractography techniques could be considered to obtain more precise locations of the tracts. For the r-fcMRI analysis, the current study was based on the seeds derived from DTI findings, future studies may engage a more exhaustive search to further identify other functional networks that might also be affected by alexithymia. Moreover, controversies on anticorrelations associated with removal of global brain signals should also be observed. Finally, the present study was correlative in nature, therefore, causal inference would have to be established in the future by lesion and prospective studies.

\section{Conclusions}

The present study confirmed the hypothesis that white matter integrity of the CC and the right SLF was correlated with the degree of alexithymia in the MDD and NC group respectively. These are also neural correlates proposed for the cognitive and affective characteristics of alexithymia based on review of existing literature. From the functional connectivity findings, we proposed that alexithymia in the MDD groups might be associated with reduced automatic emotional regulation and introspective thinking, with increased effort for cognitive regulation of emotional information; while alexithymia in NC group might be linked with reduced capacity for evaluation of emotional salience of exogenous stimuli. These findings could shed some lights on the prevailing observations that alexithymia in MDD patients is associated with the increased need for emotional regulation. They also provide additional evidence for relating alexithymia with more blunted emotional experiences in NC samples. Finally, the present study has provided neurobiological evidence to support the importance in separating healthy and clinical samples for analyses in studies of 


\section{alexithymia in the future.}

\section{Conflict of interest \\ The authors report no conflicts of interest. The authors alone are responsible for the content and writing of the paper}

\section{Acknowledgments}

This work was supported by Research Grant Council of Hong Kong General Research Fund (ref: HKU-17613815H).

\section{References}

Aboitiz, F., Scheibel, A.B., Fisher, R.S., Zaidel, E., 1992. Fiber composition of the human corpus callosum. Brain Res. 598, 143-153.

Aftanas, L., Varlamov, A., 2007. Effects of alexithymia on the activity of the anterio and posterior areas of the cortex of the right hemisphere in positive and negative emotional activation. Neurosci. Behav. Physiol. 37, 67-73.

American Psychiatric Association, 1994. Diagnostic and Statistical Manual of Mental Disorders, 4th ed. American Psychiatric Press, Washington, DC.

Aron, A.R., Robbins, T.W., Poldrack, R.A., 2004. Inhibition and the right inferior frontal cortex. Trends Cogn. Sci. 8, 170-177.

Bagby, R.M., Parker, J.D., Taylor, G.J., 1994. The twenty-item Toronto Alexithymia Scale-I. Item selection and cross-validation of the factor structure. J. Psychosom. Res. 38, 23-32.

Bankier, B., Aigner, M., Bach, M., 2001. Alexithymia in DSM-IV disorder: comparative evaluation of somatoform disorder, panic disorder, obsessive-compulsive disorder, and depression. Psychosomatics 42, 235-240.

Basser, P.J., Pierpaoli, C., 1996. Microstructural and physiological features of tissues elucidated by quantitative-diffusion-tensor MRI. J. Magn. Reson. Ser. B 111, 209-219.

Beaulieu, C, 2010. What makes diffusion anisotropic in the nervous system?. In: Jones, D.K. (Ed.), Diffusion MRI: Theory, Methods, and Applications, 1 ed. Oxford University Press, NY, pp. 92-109.

Bermond, B., Bleys, J., Stoffels, E., 2005. Left hemispheric preference and alexithymia: a neuropsychological investigation. Cogn. Emot. 19, 151-160.

Bermond, B., Clayton, K., Liberova, A., Luminet, O., Maruszewski, T., Ricci Bitti, P.E. Rimé, B., Vorst, H.H., Wagner, H., Wicherts, J., 2007. A cognitive and an affective dimension of alexithymia in six languages and seven populations. Cogn. Emot. $21,1125-1136$

Borod, J.C., Cicero, B.A., Obler, L.K., Welkowitz, J., Erhan, H.M., Santschi, C., Grunwald, I.S., Agosti, R.M., Whalen, J.R., 1998. Right hemisphere emotional perception: evidence across multiple channels. Neuropsychology 12, 446-458.

Borsci, G., Boccardi, M., Rossi, R., Rossi, G., Perez, J., Bonetti, M., Frisoni, G.B., 2009 Alexithymia in healthy women: a brain morphology study. J. Affect. Disord. 114, $208-215$.

Buckner, R.L., Snyder, A.Z., Shannon, B.J., LaRossa, G., Sachs, R., Fotenos, A.F., Sheline, Y.I., Klunk, W.E., Mathis, C.A., Morris, J.C., 2005. Molecular, structural, and functional characterization of Alzheimer's disease: evidence for a relationship between default activity, amyloid, and memory. J. Neurosci. 25, 7709-7717.

Byrne, B.M., Stewart, S.M., Lee, P.W., 2004. Validating the Beck depression inventory-II for Hong Kong community adolescents. Int. J. Test. 4, 199-216.

Chester, D.S., Pond, R.S., DeWall, C.N., 2015. Alexithymia is associated with blunted anterior cingulate response to social rejection: implications for daily rejection. Soc. Cogn. Affect. Neurosci. 10, 517-522.

Cole, J., Chaddock, C.A., Farmer, A.E, Aitchison, KJ. Simmons, A., McGuffin, P., Fu, C. H., 2012. White matter abnormalities and illness severity in major depressive disorder. Br. J. Psychiatry 201, 33-39.

Craig, A.B., 2009. How do you feel-now? The anterior insula and human awareness. Nat. Rev, Neurosci. 10, 59-70.

De Schotten, M.T., Dell'Acqua, F., Forkel, S.J., Simmons, A., Vergani, F., Murphy, D.G. Catani, M., 2011. A lateralized brain network for visuospatial attention. Nat. Neurosci. 14, 1245-1246.

Duddu, V., Isaac, M., Chaturvedi, S., 2003. Alexithymia in somatoform and depres sive disorders. J. Psychosom. Res. 54, 435-438.

Etkin, A., Egner, T., Kalisch, R., 2011. Emotional processing in anterior cingulate and medial prefrontal cortex. Trends Cogn. Sci. 15, 85-93.

Fox, M.D., Raichle, M.E., 2007. Spontaneous fluctuations in brain activity observed with functional magnetic resonance imaging. Nat. Rev. Neurosci. 8, 700-711.

Franz, M., Popp, K., Schaefer, R., Sitte, W., Schneider, C., Hardt, J., Decker, O. Braehler, E., 2008. Alexithymia in the German general population. Soc. Psychiatry Psychiatr. Epidemiol. 43, 54-62.

Greicius, M.D., Supekar, K., Menon, V., Dougherty, R.F., 2009. Resting-state functional connectivity reflects structural connectivity in the default mode network. Cereb. Cortex 19, 72-78.

Guidi, J., Fava, G.A., Picardi, A., Porcelli, P., Bellomo, A., Grandi, S., Grassi, L., Pasquini, P. Quartesan, R., Rafanelli, C, 2011. Subtyping depression in the medically ill by cluster analysis. J. Affect. Disord. 132, 383-388.

Gündel, H., López-Sala, A., Ceballos-Baumann, A.O., Deus, J., Cardoner, N., MartenMittag, B., Soriano-Mas, C., Pujol, J., 2004. Alexithymia correlates with the size of the right anterior cingulate. Psychosom. Med. 66, 132-140.
Guo, W.-b., Liu, F., Chen, J.-d., Xu, X.-j., Wu, R.-r., Ma, C.-q., Gao, K., Tan, C.-1, Sun, X... Xiao, C.-q. 2012a. Altered white matter integrity of forebrain in treatmentresistant depression: a diffusion tensor imaging study with tract-based spatial statistics. Prog. Neuropsychopharmacol. Biol. Psychiatry 38, 201-206.

Guo, W.-b., Liu, F., Xue, Z.-m., Gao, K., Wu, R.-r., Ma, C.-q., Liu, Z.-n., Xiao, C.-q., Chen, H.-f., Zhao, J.-p., 2012b. Altered white matter integrity in young adults with first-episode, treatment-naive, and treatment-responsive depression. Neurosci. Lett. 522, 139-144.

Heinzel, A., Minnerop, M., Schäfer, R., Müller, H.-W., Franz, M., Hautzel, H., 2012. Alexithymia in healthy young men: a voxel-based morphometric study. J. Affect. Disord. 136, 1252-1256.

Herbert, B.M., Herbert, C., Pollatos, O., 2011. On the relationship between interoceptive awareness and alexithymia: Is interoceptive awareness related to emotional awareness? J. Pers. 79, 1149-1175.

Hintikka, J., Honkalampi, K., Lehtonen, J., Viinamäki, H., 2001. Are alexithymia and depression distinct or overlapping constructs?: a study in a general population. Compr. Psychiatry 42, 234-239.

Ho, N.S., Lee, T.M., 2013. Alexithymia: The story of a survivor of childhood traumatic brain injury. Brain Inj. 27, 1199-1205.

Hofer, S., Frahm, J., 2006. Topography of the human corpus callosum revisitedcomprehensive fiber tractography using diffusion tensor magnetic resonance imaging. Neuroimage 32, 989-994.

Honkalampi, K., Hintikka, J., Saarinen, P., Lehtonen, J., Viinamäki, H., 2000. Is alexithymia a permanent feature in depressed patients? Results from a 6-month follow-up study. Psychother. Psychosom. 69, 303-308.

Honkalampi, K., Koivumaa-Honkanen, H., Lehto, S.M., Hintikka, J., Haatainen, K., Rissanen, T., Viinamäki, H., 2010. Is alexithymia a risk factor for major depression, personality disorder, or alcohol use disorders? A prospective populationbased study. J. Psychosom. Res. 68, 269-273.

Honkalampi, K., Saarinen, P., Hintikka, J., Virtanen, V., Viinamäki, H., 1999. Factors associated with alexithymia in patients suffering from depression. Psychother. Psychosom. 68, 270-275.

Hoppe, K.D., Bogen, J.E., 1977. Alexithymia in twelve commissurotomized patients. Psychother. Psychosom. 28, 148-155.

Ihme, K., Dannlowski, U., Lichev, V., Stuhrmann, A., Grotegerd, D., Rosenberg, N., Kugel, H., Heindel, W., Arolt, V., Kersting, A., 2013. Alexithymia is related to differences in gray matter volume: a voxel-based morphometry study. Brain Res. 1491, 60-67.

Ihme, K., Sacher, J., Lichev, V., Rosenberg, N., Kugel, H., Rufer, M., Grabe, H.-J., Pampel, A., Lepsien, J., Kersting, A., 2014. Alexithymic features and the labeling of brief emotional facial expressions-an fMRI study. Neuropsychologia 64, 289-299.

Kano, M., Fukudo, S., Gyoba, J., Kamachi, M., Tagawa, M., Mochizuki, H., Itoh, M., Hongo, M., Yanai, K., 2003. Specific brain processing of facial expressions in people with alexithymia: an H2150-PET study. Brain 126, 1474-1484.

Karlsson, H., Näätänen, P., Stenman, H., 2008. Cortical activation in alexithymia as a response to emotional stimuli. Br. J. Psychiatry 192, 32-38.

Kober, H., Barrett, L.F., Joseph, J., Bliss-Moreau, E., Lindquist, K., Wager, T.D., 2008. Functional grouping and cortical-subcortical interactions in emotion: a metaanalysis of neuroimaging studies. Neuroimage 42, 998-1031.

Kohn, N., Eickhoff, S., Scheller, M., Laird, A., Fox, P., Habel, U., 2014. Neural network of cognitive emotion regulation-an ALE meta-analysis and MACM analysis. Neuroimage 87, 345-355.

Kranczioch, C., Debener, S., Schwarzbach, J., Goebel, R., Engel, A.K., 2005. Neural correlates of conscious perception in the attentional blink. Neuroimage 24, 704-714.

Kubota, M., Miyata, J., Sasamoto, A., Kawada, R., Fujimoto, S., Tanaka, Y., Sawamoto, N., Fukuyama, H., Takahashi, H., Murai, T., 2012. Alexithymia and reduced white matter integrity in schizophrenia: a diffusion tensor imaging study on impaired emotional self-awareness. Schizophr. Res. 141, 137-143.

Lane, R., Sechrest, L., Reidel, R., Weldon, V., Kaszniak, A., Schwartz, G., 1996. Impaired verbal and nonverbal emotion recognition in alexithymia. Psychosom. Med. 58, 203-210.

Lane, R.D., Reiman, E.M., Axelrod, B., Yun, L.-S., Holmes, A., Schwartz, G.E., 1998. Neural correlates of levels of emotional awareness: evidence of an interaction between emotion and attention in the anterior cingulate cortex. J. Cogn. Neurosci. 10, 525-535.

Larsen, J.K., Brand, N., Bermond, B., Hijman, R., 2003. Cognitive and emotional characteristics of alexithymia: a review of neurobiological studies. J. Psychosom. Res. 54, 533-541.

Levant, R.F., Hall, R.J., Williams, C.M., Hasan, N.T., 2009. Gender differences in alexithymia. Psychol. Men. Masc. 10, 190-230.

Liemburg, E.J., Swart, M., Bruggeman, R., Kortekaas, R., Knegtering, H., Ćurčić-Blake, B., Aleman, A., 2012. Altered resting state connectivity of the default mode network in alexithymia. Soc. Cogn. Affect. Neurosci. 7, 660-666.

Lim, K., Helpern, J., 2002. Neuropsychiatric applications of DTI-a review. NMR Biomed. 15, 587-593.

Lin, A., Adolphs, R., Rangel, A., 2012. Social and monetary reward learning engage overlapping neural substrates. Soc. Cogn. Affect. Neurosci. 7, 274-281.

Lumley, M.A., Sielky, K., 2000. Alexithymia, gender, and hemispheric functioning. Compr. Psychiatry 41, 352-359.

Makris, N., Kennedy, D.N., McInerney, S., Sorensen, A.G., Wang, R., Caviness, V.S., Pandya, D.N., 2005. Segmentation of subcomponents within the superior longitudinal fascicle in humans: a quantitative, in vivo, DT-MRI study. Cereb. Cortex 15, 854-869.

Marchesi, C., Brusamonti, E., Maggini, C., 2000. Are alexithymia, depression, and anxiety distinct constructs in affective disorders? J. Psychosom. Res. 49, 43-49. Mattila, A.K., Salminen, J.K., Nummi, T., Joukamaa, M., 2006. Age is strongly 
associated with alexithymia in the general population. J. Psychosom. Res. 61, 629-635.

McRae, K., Reiman, E.M., Fort, C.L., Chen, K., Lane, R.D., 2008. Association between trait emotional awareness and dorsal anterior cingulate activity during emotion is arousal-dependent. Neuroimage 41, 648-655.

Menon, V., Uddin, L.Q., 2010. Saliency, switching, attention and control: a network model of insula function. Brain Struct. Funct. 214, 655-667.

Mirowsky, J., Ross, C.E., 1992. Age and depression. J. Health Soc. Behav., 187-205.

Mori, S., Wakana, S., Van Zijl, P.C., Nagae-Poetscher, L., 2005. MRI Atlas of Human White Matter. Elsevier, Amsterdam.

Nemiah, J.C., Freyberger, H., Sifneos, P.E., 1976. Alexithymia: a view of the psychosomatic process. Mod. Trends Psychosom. Med. 3, 430-439.

Nichols, T.E., Holmes, A.P., 2002. Nonparametric permutation tests for functional neuroimaging: a primer with examples. Hum. Brain Mapp. 15, 1-25.

Paradiso, S., Anderson, B.M., Boles Ponto, L.L., Tranel, D., Robinson, R.G., 2011. Altered neural activity and emotions following right middle cerebral artery stroke. J. Stroke Cerebrovasc. Dis. 20, 94-104.

Parker, J.D., Bagby, R.M., Taylor, G.J., 1991. Alexithymia and depression: distinct or overlapping constructs? Compr. Psychiatry 32, 387-394.

Parker, J.D., Keightley, M.L., Smith, C.T., Taylor, G.J., 1999. Interhemispheric transfer deficit in alexithymia: an experimental study. Psychosom. Med. 61, 464-468.

Parker, J.D., Taylor, G., Bagby, M., 1993. Alexithymia and the processing of emotional stimuli: an experimental study. New. Trends Exp. Clin. Psychiatry 9, 9-14.

Paul, L.K., 2011. Developmental malformation of the corpus callosum: a review of typical callosal development and examples of developmental disorders with callosal involvement. J. Neurodev. Disord. 3, 3-27.

Paulus, M.P., Rogalsky, C., Simmons, A., Feinstein, J.S., Stein, M.B., 2003. Increased activation in the right insula during risk-taking decision making is related to harm avoidance and neuroticism. Neuroimage 19, 1439-1448.

Paulus, M.P., Stein, M.B., 2006. An insular view of anxiety. Biol. Psychiatry 60, 383-387.

Phillips, M.L., Drevets, W.C., Rauch, S.L., Lane, R., 2003. Neurobiology of emotion perception I: the neural basis of normal emotion perception. Biol. Psychiatry 54, 504-514.

Pouga, L., Berthoz, S., de Gelder, B., Grezes, J., 2010. Individual differences in socioaffective skills influence the neural bases of fear processing: the case of alexithymia. Hum. Brain Mapp. 31, 1469-1481.

Pourtois, G., Schettino, A., Vuilleumier, P., 2013. Brain mechanisms for emotional influences on perception and attention: what is magic and what is not. Biol. Psychol. 92, 492-512.

Raven, J., 2000. The Raven's progressive matrices: change and stability over culture and time. Cogn. Psychol. 41, 1-48.

Reker, M., Ohrmann, P., Rauch, A.V., Kugel, H., Bauer, J., Dannlowski, U., Arolt, V., Heindel, W., Suslow, T., 2010. Individual differences in alexithymia and brain response to masked emotion faces. Cortex 46, 658-667.

Romei, V., De Gennaro, L., Fratello, F., Curcio, G., Ferrara, M., Pascual-Leone, A., Bertini, M., 2008. Interhemispheric transfer deficit in alexithymia: a transcranial magnetic stimulation study. Psychother. Psychosom. 77, 175-181.

Rota, G., Sitaram, R., Veit, R., Erb, M., Weiskopf, N., Dogil, G., Birbaumer, N., 2009. Self-regulation of regional cortical activity using real-time fMRI: the right inferior frontal gyrus and linguistic processing. Hum. Brain Mapp. 30, 1605-1614.

Saarijärvi, S., Salminen, J., Toikka, T., 2001. Alexithymia and depression: a 1-year follow-up study in outpatients with major depression. J. Psychosom. Res. 51, 729-733.

Salminen, J., Saarijärvi, S., Toikka, T., Kauhanen, J. Äärelä, E., 2006. Alexithymia behaves as a personality trait over a 5-year period in Finnish general population. J. Psychosom. Res. 61, 275-278.

Seghier, M.L., 2013. The angular gyrus multiple functions and multiple subdivisions. Neuroscience 19, 43-61.

Sifneos, P.E., 1973. The prevalence of "alexithymic" characteristics in psychosomatic patients. Psychother. Psychosom. 22, 255-262.

Smith, S.M., 2002. Fast robust automated brain extraction. Hum. Brain Mapp. 17, $143-155$.

Smith, S.M., Fox, P.T., Miller, K.L., Glahn, D.C., Fox, P.M., Mackay, C.E., Filippini, N., Watkins, K.E., Toro, R., Laird, A.R., 2009. Correspondence of the brain's functional architecture during activation and rest. Proc. Natl. Acad. Sci. 106, 13040-13045.

Smith, S.M., Jenkinson, M., Johansen-Berg, H., Rueckert, D., Nichols, T.E., Mackay, C. E., Watkins, K.E., Ciccarelli, O., Cader, M.Z., Matthews, P.M., 2006. Tract-based spatial statistics: voxelwise analysis of multi-subject diffusion data. Neuroimage 31, 1487-1505.

Smith, S.M., Jenkinson, M. Woolrich, M.W Beckmann, C.F, Behrens, T.E., JohansenBerg, H., Bannister, P.R., De Luca, M., Drobnjak, I., Flitney, D.E., 2004. Advances in functional and structural MR image analysis and implementation as FSL. Neuroimage 23, S208-S219.

Smith, S.M., Johansen-Berg, H., Jenkinson, M., Rueckert, D., Nichols, T.E., Miller, K.L. Robson, M.D., Jones, D.K., Klein, J.C., Bartsch, A.J., 2007. Acquisition and voxelwise analysis of multi-subject diffusion data with tract-based spatial statistics. Nat. Protoc. 2, 499-503.

Song, X.-W., Dong, Z.-Y., Long, X.-Y., Li, S.-F., Zuo, X.-N., Zhu, C.-Z., He, Y., Yan, C.-G. Zang, Y.-F., 2011. REST: a toolkit for resting-state functional magnetic resonance imaging data processing. PLoS One 6, e25031.

Sridharan, D., Levitin, D.J., Menon, V., 2008. A critical role for the right fronto-insular cortex in switching between central-executive and default-mode networks. Proc. Natl. Acad. Sci. 105, 12569-12574.

Tabibnia, G., Zaidel, E., 2005. Alexithymia, interhemispheric transfer, and right hemispheric specialization: a critical review. Psychother. Psychosom. 74, 81-92.

Taylor, G.J., Bagby, R.M., 2004. New trends in alexithymia research. Psychother. Psychosom. 73, 68-77.

Troisi, A., Belsanti, S., Bucci, A.R., Mosco, C., Sinti, F., Verucci, M., 2000. Affect regulation in alexithymia: an ethological study of displacement behavior during psychiatric interviews. J. Nerv. Ment. Dis. 188, 13-18.

Tzourio-Mazoyer, N., Landeau, B., Papathanassiou, D., Crivello, F., Etard, O., Delcroix N., Mazoyer, B., Joliot, M., 2002. Automated anatomical labeling of activations in SPM using a macroscopic anatomical parcellation of the MNI MRI single-subject brain. Neuroimage 15, 273-289.

Uddin, L.Q., Clare Kelly, A., Biswal, B.B., Xavier Castellanos, F., Milham, M.P., 2009. Functional connectivity of default mode network components: correlation, anticorrelation, and causality. Hum. Brain Mapp. 30, 625-637.

van der Velde, J., Servaas, M.N., Goerlich, K.S., Bruggeman, R., Horton, P., Costafreda S.G., Aleman, A., 2013. Neural correlates of alexithymia: a meta-analysis of emotion processing studies. Neurosci. Biobehav. Rev. 37, 1774-1785.

van der Velde, J., van Tol, M.-J., Goerlich-Dobre, K.S., Gromann, P.M., Swart, M., de Haan, L., Wiersma, D., Bruggeman, R., Krabbendam, L., Aleman, A., 2014. Dissociable morphometric profiles of the affective and cognitive dimensions of alexithymia. Cortex 54, 190-199.

Vanheule, S., Desmet, M., Verhaeghe, P., Bogaerts, S., 2007. Alexithymic depression: Evidence for a depression subtype? Psychother. Psychosom. 76, 315-316.

Vorst, H.C.M., Bermond, B., 2001. Validity and reliability of the Bermond-Vorst Alexithymia Questionnaire. Pers. Individ. Differ. 30, 413-434.

Yan, C.-G., Zang, Y.-F., 2010. DPARSF: a MATLAB toolbox for "pipeline" data analysis of resting-state fMRI. Front. Syst. Neurosci. 4, 1-13.

Yarkoni, T., Braver, T.S., 2010. Cognitive neuroscience approaches to individual differences in working memory and executive control: conceptual and methodological issues, Handbook of Individual Differences in Cognition. Springer pp. 87-107.

Zhu, X., Yi, J., Yao, S., Ryder, A.G., Taylor, G.J., Bagby, R.M., 2007. Cross-cultural validation of a Chinese translation of the 20-item Toronto Alexithymia Scale. Compr. Psychiatry 48, 489-496. 